\title{
First Measurement of the Rate $K_{L}^{0} \rightarrow \pi \mu \nu \gamma$
}

\author{
M. Bender, I. Augustin, M. Holder, I. Schwarze, M. Ziolkowski \\ Fachbereich Physik, Universität Siegen, D-57068 Siegen ${ }^{1}$, Germany \\ V. Fanti, A. Lai, L. Musa, A. Nappi, M.G. Setzu \\ Dipartimento di Fisica dell'Università e Sezione dell'INFN di Cagliari, I-09100 Cagliari, \\ Italy \\ R.W. Moore ${ }^{2}$, D.J. Munday, M.A. Parker, S.F. Takach ${ }^{3}$, T.O. White \\ HEP, Cavendish Lab., Physics Department, University of Cambridge, GB-Cambridge \\ CBз OHE, UK \\ G. Barr, G. Bocquet, J. Bremer, A. Ceccucci ${ }^{4}$, J. Cogan, D. Cundy, N. Doble, \\ G. Fischer, W. Funk, L. Gatignon, A. Gianoli, A. Gonidec, P. Grafstrom, \\ W. Kubischta, V. Marzulli, G. Kesseler, J.P. Matheys, A. Norton, S. Palestini ${ }^{4}$, \\ B. Panzer-Steindel, D. Schinzel, H. Taureg, M. Velasco, O. Vossnack \\ CERN, CH-1211 Geneva 23, Switzerland \\ V. Kekelidze, I. Kojevnikov, I. Potrebenikov, G. Tatichvili, A. Tkatchev \\ Joint Institute for Nuclear Research, Dubna, Russian Federation \\ L. Bertolotto, O. Boyle, B. Hay ${ }^{5}$, N.E. Mc Kay, G. Oakland, H. Parsons, \\ K.J. Peach, C. Talamonti, A. Walker \\ Physics Department, University of Edinburgh, GB-Edinburgh EH9 3JZ, UK \\ P. Dalpiaz, J. Duclos, A. Formica, P.L. Frabetti, M. Martini, F. Petrucci, \\ M. Porcu, F. Rossi, M. Savrie, C. Simani \\ Dipartimento di Fisica dell'Università e Sezione dell'INFN di Ferrara, I-44100 Ferrara, \\ Italy
}

A. Bizzeti, M. Calvetti, M. Lenti, A. Michetti

Dipartimento di Fisica dell'Università e Sezione dell'INFN di Firenze, I-50125 Firenze, Italy

\footnotetext{
${ }^{1}$ Funded by the German Federal Minister for Research and Technology (BMBF) under contract 056SI74

${ }^{2}$ Present address: Department of Physics and Astronomy, Michigan State University, East Lansing, MI 48824-1116, USA

${ }^{3}$ Present address: Department of Physics, Wayne State University, Detroit, MI 48201, USA.

${ }^{4}$ Permanent address, Dipartimento di Fisica Sperimentale dell'Università e Sezione dell'INFN di Torino, I-10125 Torino, Italy

${ }^{5}$ Present address: Cavendish Laboratory, University of Cambridge, Cambridge CB3 0HE, UK.
} 
H.G. Becker, T. Beier, H. Blümer ${ }^{6}$, P. Buchholz ${ }^{7}$, D. Coward $^{8}$, C. Ebersberger, A. Kalter, K. Kleinknecht, U. Koch, L. Köpke, F. Leber, S. Luitz ${ }^{6}$, B. Renk, J. Scheidt, J. Schmidt, V. Schönharting, R. Wilhelm, O. Zeitnitz Institut für Physik, Universität Mainz, D-55099 Mainz' ${ }^{9}$, Germany

J.C. Chollet, S.Crépé, L. Fayard, L. Iconomidou-Fayard, J. Ocariz ${ }^{10}$, G. Unal Laboratoire de l'Accélérateur Linéaire, IN2P3-CNRS, Université de Paris-Sud, F-91406 Orsay $^{11}$, France

G. Anzivino, P. Cenci, P. Lubrano, M. Pepe

Dipartimento di Fisica dell'Università e Sezione dell'INFN di Perugia, I-06100 Perugia, Italy

P. Calafiura, R. Carosi, R. Casali, C. Cerri, F. Costantini, R. Fantechi, S. Giudici, B. Gorini, I. Mannelli, G. Pierazzini, M. Sozzi

Dipartimento di Fisica dell'Università, Scuola Normale Superiore e Sezione INFN di Pisa, I-56100 Pisa, Italy

J.B. Cheze, M. De Beer, P. Debu, P. Hristov, E. Mazzucato, B. Peyaud, S. Schanne, O. Steinkamp ${ }^{12}$, R. Turlay, B. Vallage CEA-Saclay-DSM/DAPNIA, F-91191 Gif-sur-Yvette, France

R. Arcidiacono, C. Biino, G. Govi, P. Maas ${ }^{13}$, F. Marchetto, E. Menichetti, Dipartimento di Fisica Sperimentale dell'Università e Sezione dell'INFN di Torino, I-10125 Torino, Italy

H. Dibon, M. Jeitler, M. Markytan, I. Mikulec, G. Neuhofer, M. Pernicka, A. Taurok

Österreichische Akademie der Wissenschaften, Institut für Hochenergiephysik, 1050 Wien, Austria

Submitted to Physics Letters B

\footnotetext{
${ }^{6}$ Present address CERN, CH-1211 Geneva 23, Switzerland

${ }^{7}$ Present address: Institut für Physik, Universität Dortmund. D-44221 Dortmund, Germany

${ }^{8}$ Present address: SLAC, Stanford, CA 94309, USA

${ }^{9}$ Funded by the German Federal Minister for Research and Technology (BMBF) under contract $7 \mathrm{MZ} 18 \mathrm{P}(4)-\mathrm{TP} 2$

${ }^{10}$ Permanent address: Departamento de Fíisica, Universidad de los Andes, Mérida 5101-A, Venezuela

${ }^{11}$ Funded by Institut National de Physique des Particules et de Physique Nucléaire (IN2P3), France

${ }^{12}$ Present address NIKHEF, 1009 DB Amsterdam, Netherlands

${ }^{13}$ Present address Dept. of Physics, Northwestern University, Evanston, IL, USA
} 


\begin{abstract}
In a preliminary version of the NA48 experiment at the CERN SPS, in which an iron- scintillator sandwich calorimeter was used as a photon detector, the ratio of $\mathrm{K}_{L} \rightarrow \pi \mu \nu \gamma$ and $\mathrm{K}_{L} \rightarrow \pi \mu \nu$ decays was measured. The branching ratio $\mathrm{BR}\left(\mathrm{K}_{L} \rightarrow \pi \mu \nu \gamma, \mathrm{E}_{\gamma}^{*} \geq 30 \mathrm{MeV}\right) /\left(\mathrm{K}_{L} \rightarrow \pi \mu \nu\right)$ is found to be $\left(2.08 \pm 0.17_{-0.21}^{+0.16}\right) \times 10^{-3}$, where the first error is statistical and the second systematic. The result is in agreement with theoretical predictions.
\end{abstract}




\section{Introduction}

Radiative decays of kaons, when compared to nonradiative decays, provide an interesting key to hadronic structure. The photon carries information on the process in which it is emitted. The dominant contribution in semileptonic radiative decays (fig. 1a,b) comes from the radiation of the final state charged particles. This is known as internal bremsstrahlung (IB). There is also a small contribution due to emission from intermediate hadronic states, the structure dependent radiation (SD) (fig. 1c). The relative size of these effects can be calculated in models of the strong interaction. Following early estimates, based on current algebra $[1,2,3,4]$, calculations in the framework of chiral perturbation theory have been made recently [5]. Experiments on radiative Ke3 decays $[6,7]$ are in agreement with predictions, but are mainly sensitive to internal bremsstrahlung. In radiative $\mathrm{K} \mu 3$ decays the IB contribution is reduced because of the larger lepton mass; the decay is therefore more sensitive to the internal structure effects. The predictions for the branching ratios are $\mathrm{BR}\left(\mathrm{K}_{L}^{0} \rightarrow \pi \mu \nu \gamma, \mathrm{E}_{\gamma}^{*} \geq 30 \mathrm{MeV}\right) /\left(\mathrm{K}_{L} \rightarrow \pi \mu \nu\right)=2.17 \times 10^{-3}$ [2] if structure dependent terms are neglected, and $\mathrm{BR}\left(\mathrm{K}_{L}^{0} \rightarrow \pi \mu \nu \gamma, \mathrm{E}_{\gamma}^{*} \geq 30 \mathrm{MeV}, \theta_{\mu \gamma}^{*}>\right.$ $\left.20^{\circ}\right) /\left(\mathrm{K}_{L} \rightarrow \pi \mu \nu\right)=2.06 \times 10^{-3}[5]$ in a different theoretical approach where structure effects are included as part of a perturbation expansion. Structure dependent effects are expected at a level of a few percent of the rate.

We report here on the first measurement of the radiative $\mathrm{K} \mu 3$ decay. The experiment was performed with an early version of the NA48 detector, designed for a measurement of $\epsilon^{\prime} / \epsilon$, in a neutral kaon beam at the CERN SPS.

\section{Experimental Setup and Data Taking}

The neutral kaon beam is derived at $2.4 \mathrm{mrad}$ from a Be target hit by $450 \mathrm{GeV}$ protons. It is confined by three collimators to a cone of $\pm 0.15 \mathrm{mrad}$ opening angle; the final collimator is located at $125 \mathrm{~m}$ from the target. Decays of kaons in an $80 \mathrm{~m}$ long space downstream of the final collimator are recorded in the subsequent detector, which consists of a magnetic spectrometer, a scintillation counter hodoscope, an iron/scintillator sandwich calorimeter, and three planes of muon counters. The neutral beam traverses the detector in an evacuated vacuum pipe of $16 \mathrm{~cm}$ diameter. The detector elements extend to a radius of about $120 \mathrm{~cm}$. Various rings of counters surround the decay volume; they are used to tag charged particles and photons which escape the detector. A more detailed description of the NA48 experiment can be found in [8].

The detector properties relevant for the present analysis can be summarized as follows: 
- The magnetic spectrometer of $20 \mathrm{~m}$ total length consists of two drift chambers in front and one following a central dipole magnet operated to give a $267 \mathrm{MeV} / \mathrm{c}$ transverse momentum kick. Each drift chamber is composed of four double planes with staggered wires to resolve left-right ambiguities. The wire orientations in the four views are horizontal, vertical, and $\pm 45^{\circ}$ to the horizontal plane. The resolution of a coordinate in the drift chamber is $110 \mu \mathrm{m}$, the momentum resolution $\Delta \mathrm{p} / \mathrm{p}=0.6 \%$ for an average momentum of $45 \mathrm{GeV} / \mathrm{c}$.

- A scintillator hodoscope is located in front of the calorimeter and used to trigger on charged particles.

- The iron/scintillator-sandwich calorimeter has $12 \mathrm{~cm}$ wide scintillation counter strips, oriented horizontally or vertically in successive planes. The planes are separated by $2.5 \mathrm{~cm}$ of iron. The light guides of counters with the same orientation but at different depths are combined such that the calorimeter appears as one plane of horizontal and one plane of vertical counters. Both these planes, centered on the beam, are divided into four quadrants. The calorimeter is longitudinally segmented in two parts, each with a total length of $60 \mathrm{~cm}$ of iron. Only the first part is relevant for the measurement of photons. The time resolution of the calorimeter was given by an effective gate width of $150 \mathrm{~ns}$ in which the integrated charge in each channel was measured. The calorimeter was calibrated with electrons from Ke3 decays between $3 \mathrm{GeV}$ and $30 \mathrm{GeV}$; the energy resolution was found to be $\Delta \mathrm{E} / \mathrm{E}=(32 \pm 5) \% / \sqrt{\mathrm{E}}$ in the interval $3 \mathrm{GeV}<\mathrm{E}<7 \mathrm{GeV}$ most relevant for the present analysis. The indicated error covers statistical and systematical uncertainties.

- The muon counters have horizontal strips of $25 \mathrm{~cm}$ width in the first plane, vertical strips of the same width in the second plane and $45 \mathrm{~cm}$ wide horizontal strips in the third plane. The planes are mutually separated by $80 \mathrm{~cm}$ of iron. The presence of a pulse above a discriminator threshold is recorded in each strip of the first two planes. Only the logical OR of all hits is available for the third plane.

- The time of the logical OR of the veto counters surrounding the decay volume is measured with $0.5 \mathrm{~ns}$ resolution.

Data were collected during one day of running in 1995 with different concurrent trigger conditions. For the K $\mu 3 \gamma$ trigger at least two particles in the scintillator hodoscope, coincident hits in the first two muon counter planes and more than $8 \mathrm{GeV}$ energy deposition in the calorimeter were required. Additional triggers were a random trigger, the rate of which was proportional to beam intensity as monitored by scintillation counters in the beam, and a minimum bias trigger in which the muon counter condition of the K $\mu 3 \gamma$ trigger was dropped. This latter trigger was used for a determination of the muon counter efficiencies. The $\mathrm{K} \mu 3 \gamma$ decay is normalized to 
the $\mathrm{K} \mu 3$ decay, taken with the same trigger condition. The average beam intensity was about $5 \%$ of the expected NA48 beam intensity, with $7.8 \times 10^{10}$ protons per pulse on target. The data sample collected with this running condition, at an average of 4500 triggers per burst, consists of $12.3 \times 10^{6}$ events with the K $\mu 3$ trigger and $1.2 \times 10^{6}$ minimum bias events.

\section{Data analysis}

\subsection{Event selection}

Events are selected in which two tracks from a common vertex are reconstructed in the magnetic spectrometer. In order to avoid possible inefficiencies at the geometrical boundaries of the detector or cluster reconstruction problems in the calorimeter, a set of further cuts is imposed.

- The z-position $\mathrm{V}_{Z}$ of the vertex is between $5 \mathrm{~m}$ and $70 \mathrm{~m}$ from the final collimator.

- The distance between the beam axis and the track position in the first chamber of the spectrometer is at least $15 \mathrm{~cm}$ and less than $120 \mathrm{~cm}$. At the longitudinal position of the calorimeter, the distance from the tracks to the beam axis has to be between $26.4 \mathrm{~cm}$ and $117.8 \mathrm{~cm}$. Both tracks are contained throughout the detector inside an octagon of $2.4 \mathrm{~m}$ inscribed diameter.

- The momentum of each of the charged particles is above $10 \mathrm{GeV} / \mathrm{c}$ and the sum of their momenta is above $30 \mathrm{GeV} / \mathrm{c}$.

- The muon is identified by an energy deposition of less than $4.5 \mathrm{GeV}$ in both the front and back module of the calorimeter. If both particles either satisfy or fail this condition, the event is rejected.

- A photon is accepted if its energy is above $3 \mathrm{GeV}$ and the energy found in the back module of the calorimeter is less than $1 \mathrm{GeV}$. The shower width must be smaller than $12 \mathrm{~cm}$, the shower position has to be in the range $22.6 \mathrm{~cm}<|x|<$ $117.8 \mathrm{~cm}$ and $22.6 \mathrm{~cm}<|y|<117.8 \mathrm{~cm}$. The distance between photon and muon impacts in the calorimeter has to be at least $18 \mathrm{~cm}$ in both, horizontal and vertical projections, if both the photon and the muon are in the same quadrant. The distance between photon and pion impact points is at least $80 \mathrm{~cm}$.

- There is no hit in the veto rings within 150 ns preceeding and following the event and no additional space point in the drift chambers from -175 ns to $+5 \mathrm{~ns}$. 
- The vertical muon counter strip centered at $\mathrm{x}=24.5 \mathrm{~cm}$ was found to be only $\approx 50 \%$ efficient. Therefore, all events with hits in this strip were rejected during reconstruction.

After these cuts, $1.36 \times 10^{6} \mathrm{~K} \mu 3$ events and $4261 \mathrm{~K} \mu 3 \gamma$ candidates remain.

\subsection{Background}

Background to $K \mu 3 \gamma$ decays is expected from several sources.

\subsection{1 $K_{L} \rightarrow \pi^{+} \pi^{-} \pi^{0}$ decays (referred to as $\mathrm{K} 3 \pi$ )}

These contribute to the $\mathrm{K} \mu 3 \gamma$ sample if one of the pions decays and one of the photons from $\pi^{0}$ decay escapes the detector. A convenient way to suppress this background is to cut in the $\mathrm{p}_{0}^{\prime 2}(\pi \mu)$ variable [9], which, calculated in the present analysis under the hypothesis of a $\mathrm{K} \mu 3$ decay, is the square of the kaon momentum in a frame boosted along the $K_{L}$ direction such that the sum of the longitudinal momenta of the charged particles is zero. In a Monte Carlo simulation, including reconstruction errors, a cut $\mathrm{p}_{0}^{\prime 2}(\pi \mu)<0.025(\mathrm{GeV} / \mathrm{c})^{2}$ eliminates all but $0.04 \%$ of $\mathrm{K} 3 \pi$ decays, whereas only $20.7 \%$ of $\mathrm{K} \mu 3 \gamma$ are lost (see fig. 2). After this cut, $1.24 \times 10^{6}$ $\mathrm{K} \mu 3-$ and $1133 \mathrm{~K} \mu 3 \gamma$-candidates are left.

\subsection{2 $K_{L} \rightarrow \pi e \nu(\gamma)$ decays}

These decays contribute to the $K_{L} \rightarrow \pi \mu \nu(\gamma)$ channels if the pion decays and the electron is misidentified as a pion. Electrons can be distinguished from pions by their different shower development in the calorimeter. For an electron, the energy $\mathrm{E}$ in the front module of the calorimeter agrees with the momentum $\mathrm{p}$ measured in the spectrometer, and the shower is narrow. Fig. 3 shows the distribution in shower width $\sigma$ and $\mathrm{E} / \mathrm{p}$ for a sample of electrons, pions and the track not identified as muon in a sample of $\mathrm{K} \mu 3$ candidates. A cut at $\mathrm{E} / \mathrm{p}=0.75$ and $\sigma=10.6 \mathrm{~cm}$, as indicated, eliminates $94.4 \%$ of the electrons and only $14 \%$ of the $\mathrm{K} \mu 3(\gamma)$ events. After this cut a sample of $1.006 \times 10^{6} \mathrm{~K} \mu 3$ events and $945 \mathrm{~K} \mu 3 \gamma$ candidates remains.

\subsection{3 $K_{L} \rightarrow \pi \mu \nu$ decays with an accidental photon}

This background depends on beam intensity and on the time resolution of the calorimeter. It is measured by recording random triggers, at a rate proportional to beam intensity. The random events are then superimposed on normal triggers at the raw data level by software, and events are reconstructed with the standard algorithm. The accidental activity is artificially doubled in this way. The difference 
in rate between these overlaid events and the candidate events is taken as a measure of the accidental effect in the candidate events. The error of this linear approximation is small since the effect itself is small. The ratio $(\mathrm{K} \mu 3+$ accidental $\gamma) / \mathrm{K} \mu 3$ is $\left(0.052 \pm 0.004_{\text {stat. }}\right) \times 10^{-3}$.

2.2.4 $K_{L} \rightarrow \pi \mu \nu$ decays with a fake photon generated in the pion shower development

The measurement of photons in an unsegmented hadron calorimeter is affected by background from neutrons in the hadron shower which deposit their energy at some distance from the shower core and create an isolated cluster of energy which is misinterpreted as an isolated photon. These fake photons from shower fluctuations have an azimuthally symmetric distribution around the pion direction. The real photons from $\mathrm{K} \mu 3 \gamma$ decays are emitted by both pions and muons. Seen from the pion they are expected to be found rather in the direction of the muon than opposite to it (fig. 4) This difference in distributions of real and fake photons allows a separation of the two components and an estimate of the remaining background. The observed background was parametrized as a function of the photon energy and the distance between pion and photon. A suitable cut for the separation of signal and background will be discussed below.

\section{Results}

It is convenient to display the signal and the remaining background in the variable $\mathrm{p}_{0}^{\prime 2}(\pi \mu \gamma)$, which is the square of the kaon momentum in a system where the sum of longitudinal momenta of pion, muon and photon vanishes under the hypothesis of a $\mathrm{K} \mu 3 \gamma$ decay. The distribution of the $945 \mathrm{~K} \mu 3 \gamma$ candidates in this variable is shown in fig. 5. The signal appears mainly at positive $\mathrm{p}_{0}^{\prime 2}(\pi \mu \gamma)$, in contrast to the background. 275 events of background due to accidental photons and shower fluctuations are kinematically incompatible with $\mathrm{K} \mu 3 \gamma$ decays and can therefore be rejected. The photon energy distributions in the laboratory and the centre of mass system for the remaining $\mathrm{K} \mu 3 \gamma$ candidates are shown in fig. 6 . In the transformation to the kaon centre of mass system there is an ambiguity due to the unmeasured neutrino. There are two solutions, one in which the neutrino goes forward and one in which it goes backward in the centre of mass system. The proper weight of these solutions, considering the acceptance of the detector, is determined by a Monte Carlo calculation. For this calculation a knowledge of the kaon spectrum is necessary. The spectrum was determined from K $\mu 3$ decays by an iterative method $[10,11]$. 
Among the 670 events with photon energies above $3 \mathrm{GeV}, 85$ are due to accidental photons and 205 due to pion shower fluctuations. After background subtraction and a final cut on kaon energy, $40 \mathrm{GeV}<\mathrm{E}_{K_{L}}<150 \mathrm{GeV}$, a signal of $363 \mathrm{~K} \mu 3 \gamma$ events is left. Among these, 252 events have photons with centre of mass energies of more than $30 \mathrm{MeV}$. This is the final sample to be normalized to $934700 \mathrm{~K} \mu 3$ events.

For acceptance calculations, $6 \times 10^{6} \mathrm{~K} \mu 3 \gamma$ events and $4 \times 10^{6} \mathrm{~K} \mu 3$ events have been generated using MonteCarlo simulations based on the formula given in [2, 12]. The acceptances were determined to be $(2.497 \pm 0.015) \%$ for K $\mu 3 \gamma$ events and $(19.29 \pm 0.03) \%$ for $\mathrm{K} \mu 3$ events.

Systematic uncertainties arise from the precision of the energy calibration in the calorimeter, the background subtraction and the acceptance calculations. They have been estimated by a variation of those parameters which influence most the acceptance and the signal/background ratio. Table 1 gives an overview of the varied parameters and their effect on the measured branching ratio.

\begin{tabular}{|l|r|r|}
\hline changed parameter & variation & $\Delta \mathrm{BR} / \mathrm{BR}$ \\
\hline \hline HAC energy calibration & $+60 \mathrm{MeV}$ & $+3.2 \%$ \\
& $-60 \mathrm{MeV}$ & $-3.1 \%$ \\
\hline minimum distance $\pi-\gamma$ & $80 \mathrm{~cm} \rightarrow 72 \mathrm{~cm}$ & $-4.7 \%$ \\
& $80 \mathrm{~cm} \rightarrow 88 \mathrm{~cm}$ & $-8.4 \%$ \\
\hline minimum $_{\gamma}$ & $3 \mathrm{GeV} \rightarrow 2.5 \mathrm{GeV}$ & $-1.2 \%$ \\
& $3 \mathrm{GeV} \rightarrow 3.5 \mathrm{GeV}$ & $+4.6 \%$ \\
\hline $\mathrm{p}_{0}^{\prime 2}(\pi \mu)$-cut & $0.025(\mathrm{GeV} / \mathrm{c})^{2} \rightarrow 0.020(\mathrm{GeV} / \mathrm{c})^{2}$ & $-3.2 \%$ \\
\hline$\gamma$-position in HAC & $|x, y|<117.8 \mathrm{~cm} \rightarrow|x, y|<106.0 \mathrm{~cm}$ & $+5.4 \%$ \\
& $|x, y|>22.6 \mathrm{~cm} \rightarrow|x, y|>34.4 \mathrm{~cm}$ & $-0.1 \%$ \\
\hline
\end{tabular}

Table 1:

The final result for the branching ratio is:

$$
\operatorname{BR}\left(\frac{\left.K_{L} \rightarrow \pi \mu \nu \gamma, E_{\gamma}^{*} \geq 30 M e V\right)}{K_{L} \rightarrow \pi \mu \nu}\right)=\left(2.08 \pm 0.17_{\text {stat. }-0.21 \text { syst. }}^{+0.16}\right) \times 10^{-3}
$$

This is in agreement with the theoretical prediction from [2]:

$$
\operatorname{BR}\left(\frac{\left.K_{L} \rightarrow \pi \mu \nu \gamma, E_{\gamma}^{*} \geq 30 M e V\right)}{K_{L} \rightarrow \pi \mu \nu}\right)=2.17 \times 10^{-3}
$$


With an additional cut $\theta_{\mu \gamma}^{*}>20^{\circ}, 1.4$ events are lost after background subtraction. The acceptance is recalculated and one obtains

$$
\operatorname{BR}\left(\frac{K_{L} \rightarrow \pi \mu \nu \gamma, E_{\gamma}^{*} \geq 30 M e V, \theta_{\mu \gamma}^{*}>20^{\circ}}{K_{L} \rightarrow \pi \mu \nu}\right)=\left(2.06 \pm 0.17_{\text {stat. }{ }_{-0.20 \text { syst. }}}^{+0.16}\right) \times 10^{-3}
$$

in good agreement with the prediction from chiral perturbation theory [5]:

$$
\operatorname{BR}\left(\frac{K_{L} \rightarrow \pi \mu \nu \gamma, E_{\gamma}^{*} \geq 30 M e V, \theta_{\mu \gamma}^{*}>20^{\circ}}{K_{L} \rightarrow \pi \mu \nu}\right)=2.06 \times 10^{-3}
$$

In conclusion, this first measurement of the radiative $\mathrm{K} \mu 3$ decay confirms the predictions, but is not yet sufficiently accurate to distinguish between different theoretical treatments of hadron structure effects or even to establish such an effect.

\section{Acknowledgements}

We would like to thank the technical staff of the participating Laboratories, Universities and affiliated computing centres for their efforts in the construction and operation of the detector during the first year of data taking. The Cambridge and Edinburgh groups thank the UK Particle Physics and Astronomy Research Council for financial support. 


\section{References}

[1] E. Fischbach and J. Smith, Phys. Rev. 184 (1969) 1645

[2] H.W. Fearing et al., Phys. Rev. D2 (1970) 542

[3] H.W. Fearing et al., Phys. Rev. Lett. 24 (1970) 189

[4] M.G. Doncel, Phys. Lett. 32B (1970) 623

[5] J. Bijnens et al.: Nucl. Phys. B396 (1993) 81

[6] K.J. Peach et al., Phys. Lett. 35B (1971) 351

[7] F. Leber et al.: Phys. Lett. B 369 (1996) 69

[8] I. Augustin, Proceedings of the 28th International Conference on High Energy Physics, Warsaw, Poland, 1996, Editors: Z. Ajduk and A.K. Wroblewski, World Scientific, p. 1679

[9] D. Luers et al.: Phys. Rev. 133 (1964) B1276

[10] A. Böhm et al.: Nucl. Phys. B9 (1969) 605

[11] A. Kreutz et al.: Zeitschrift für Physik C 65, 67-74 (1995)

[12] Review of Particle Physics, Phys. Rev. D51,1 (1996) 415 


\section{Figure captions}

Fig. 1: Diagrams for internal Bremsstrahlung and the structure dependent contribution to the radiative $\mathrm{Kl} 3$ decay.

Fig. 2: Distribution of expected signal and background from $\mathrm{K}_{L} \rightarrow \pi^{+} \pi^{-} \pi^{0}$ decays in the variable $\mathrm{p}_{0}^{\prime 2}(\pi \mu)$.

Fig. 3: Measured ratio E/p and shower width $\sigma$ for:
a) Electron sample
b) Pion sample
c) the track not identified as muon in the $\mathrm{K} \mu 3$ candidate sample

Fig. 4: a) Position of the fake photon with respect to the $\pi$-shower for those $\mathrm{K} \mu 3 \gamma$ candidates which are kinematically incompatible with a $\mathrm{K} \mu 3 \gamma$ decay. The $\pi$-shower is located at $(0,0)$, the $\mathrm{X}$-axis is defined by the line $\pi-\mu$.

b) Position of real photons for simulated $\mathrm{K} \mu 3 \gamma$ events in the frame defined in a)

c) Position of accidental photons in the frame defined in a)

For the final sample only photons with a distance of more than $80 \mathrm{~cm}$ to the pion impact are accepted (see text)

Fig. 5: Distribution of signal and background in the variable $\mathrm{p}_{0}^{\prime 2}(\pi \mu \gamma)$ :

a) Comparison of background and expected signal

b) Comparison of data and the sum of background plus expected signal

Fig. 6: a) Photon energy spectrum in the laboratory frame for $\mathrm{K} \mu 3 \gamma$ candidates.

b) Photon energy in the $K_{L}$ CM-system. Only events with photons of $\mathrm{E}_{\gamma} \geq 3 \mathrm{GeV}$ in the laboratory frame are accepted and transformed into the $K_{L}$ CM-system. 

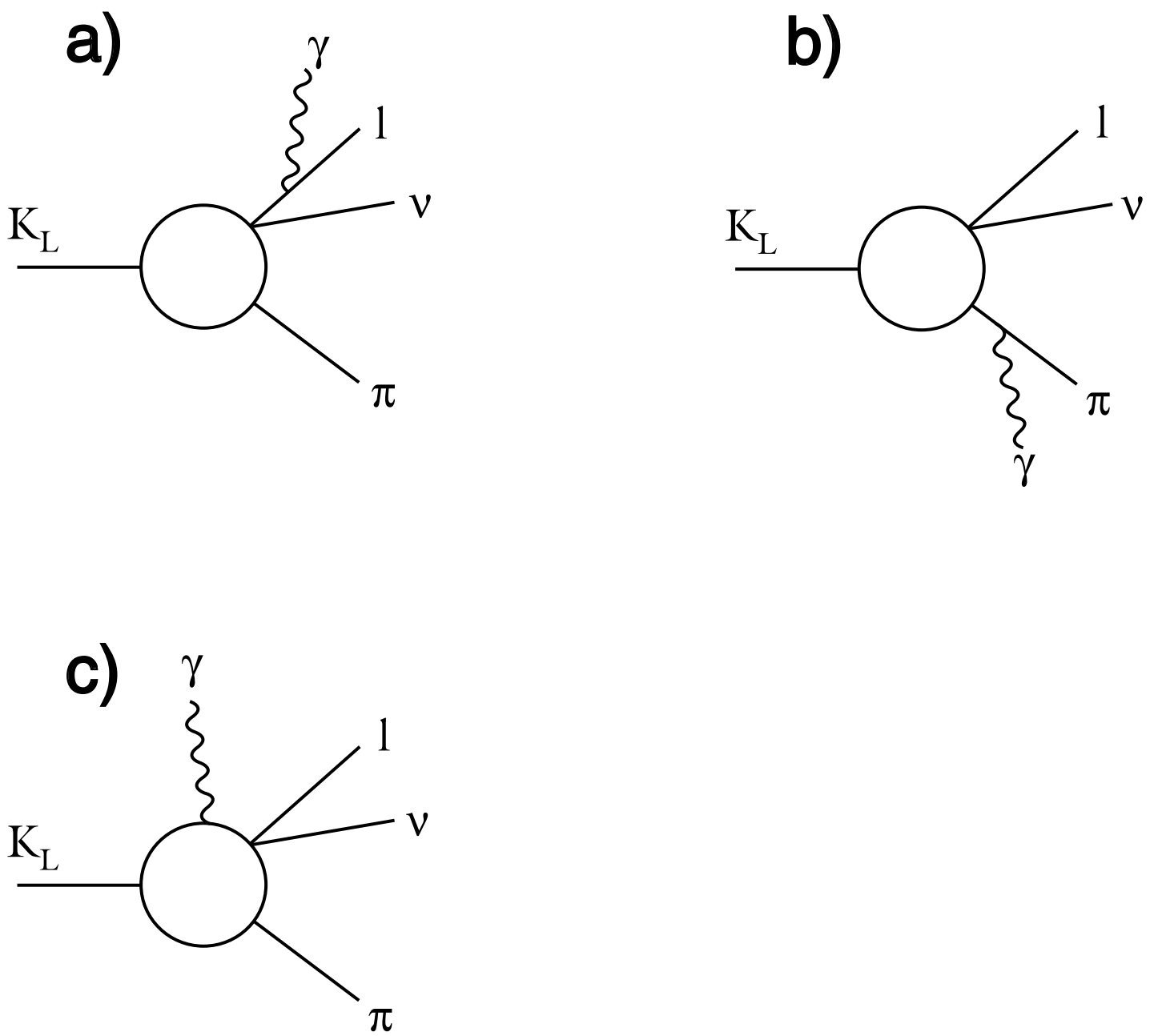

Fig. 1 


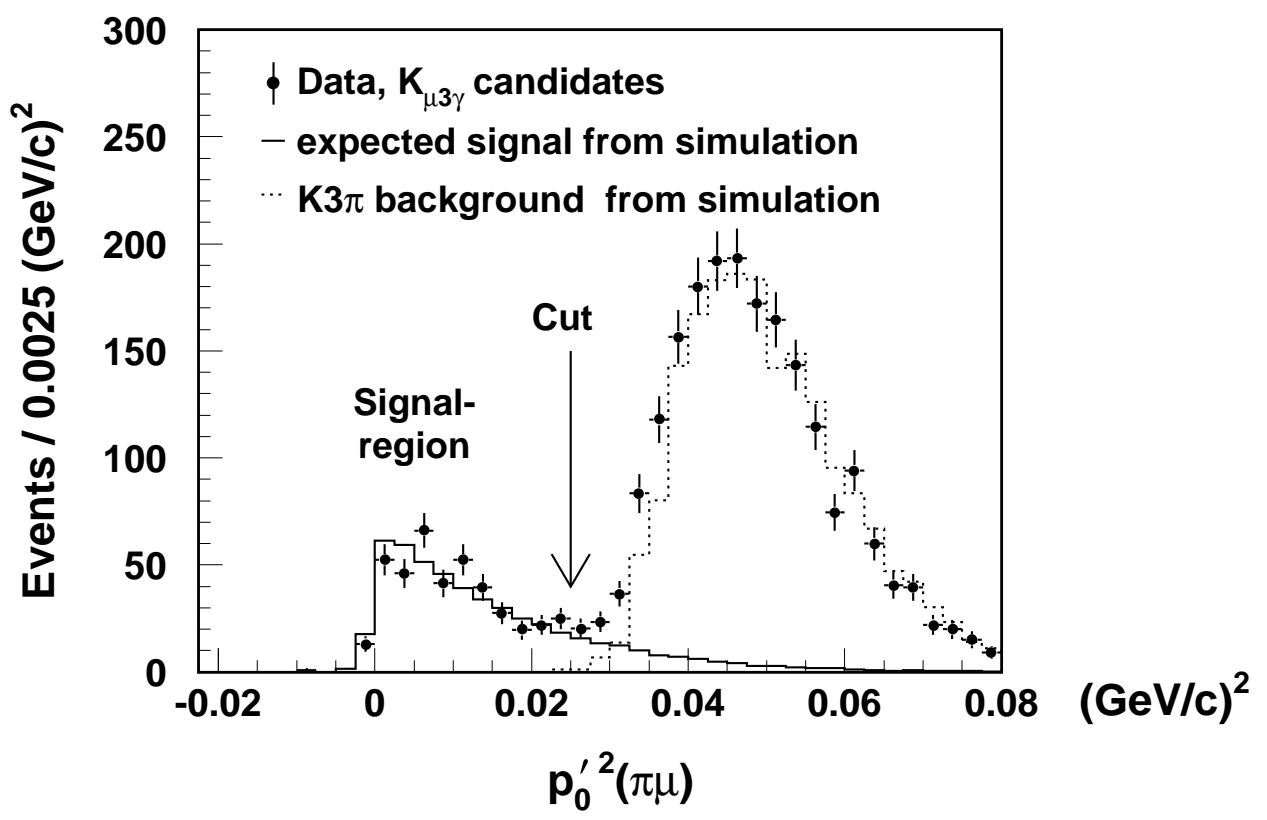

Fig. 2 
a)

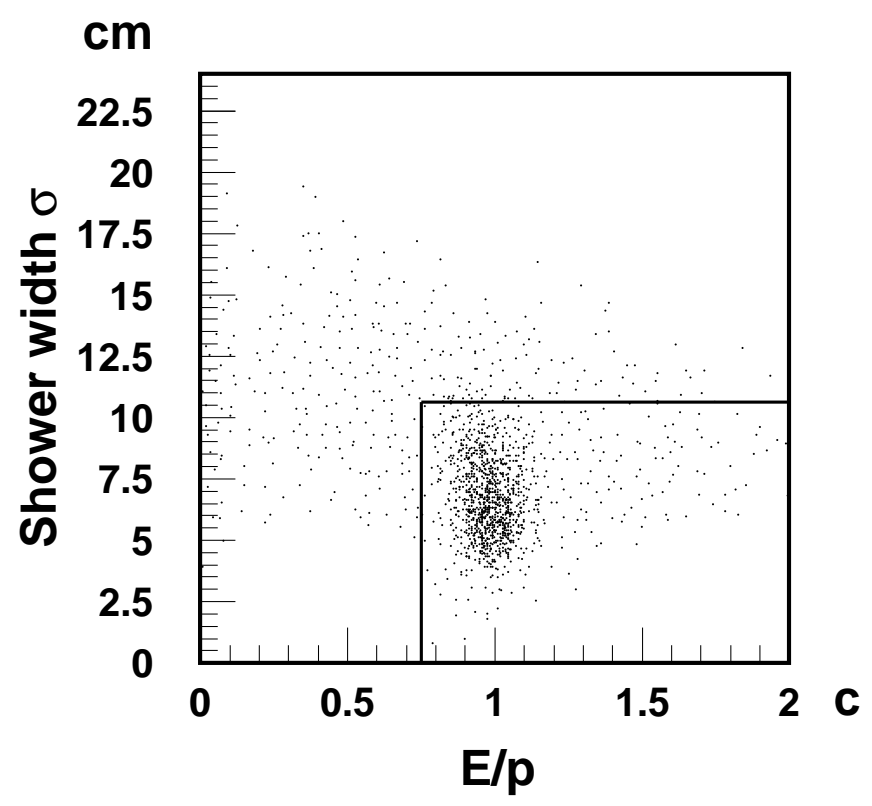

b)

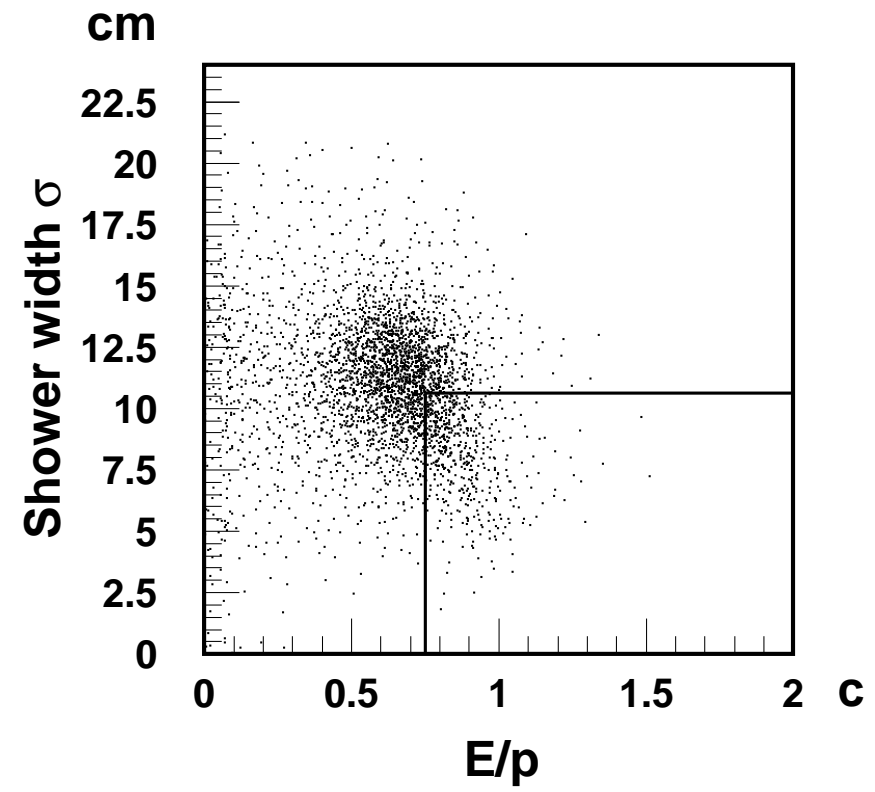

Fig. 3 
c)

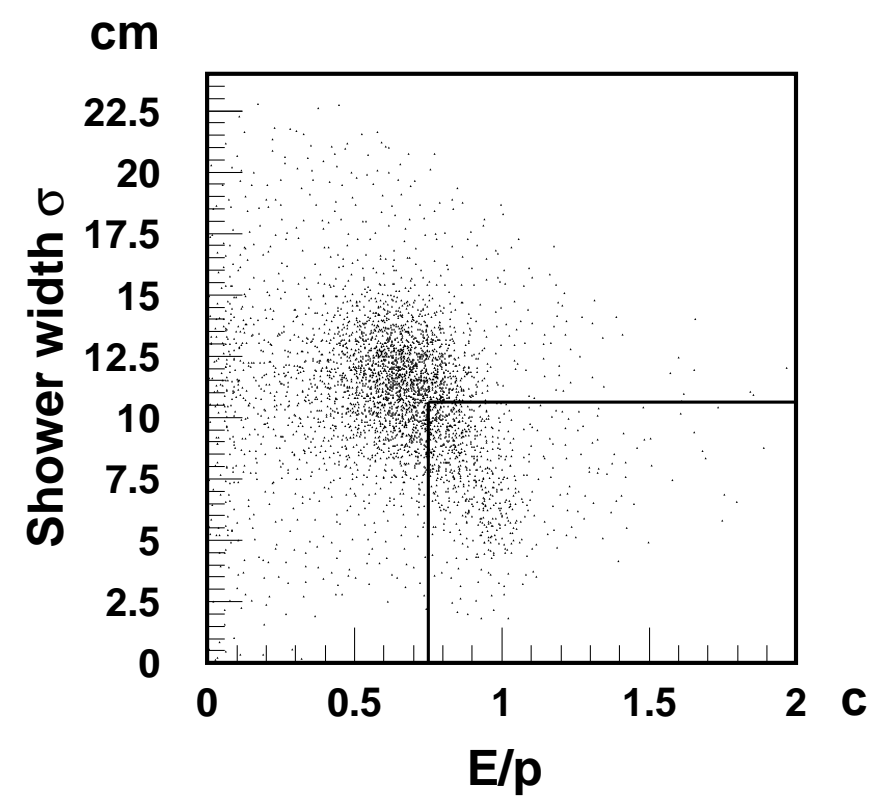

Fig. 3 
a)

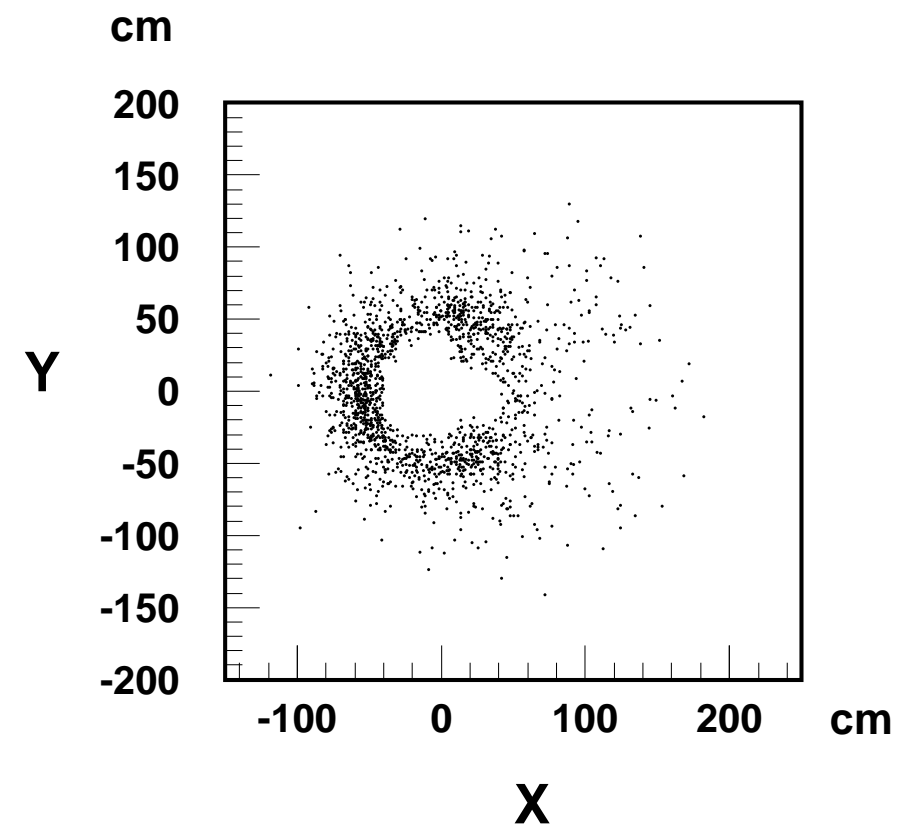

b)

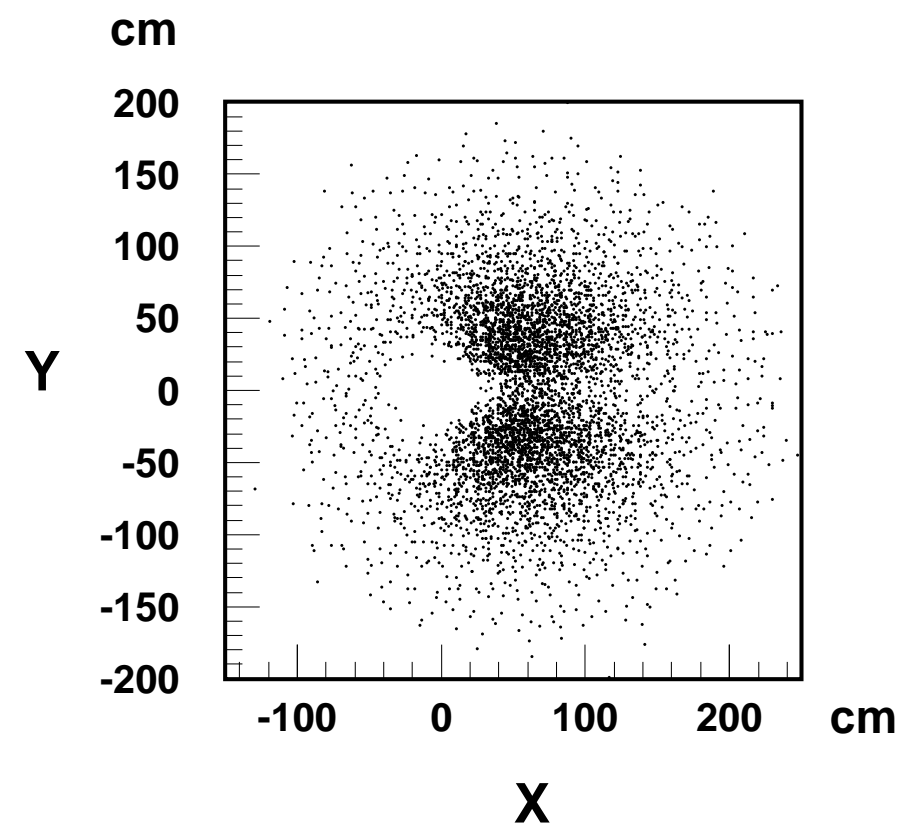

Fig. 4 
c)

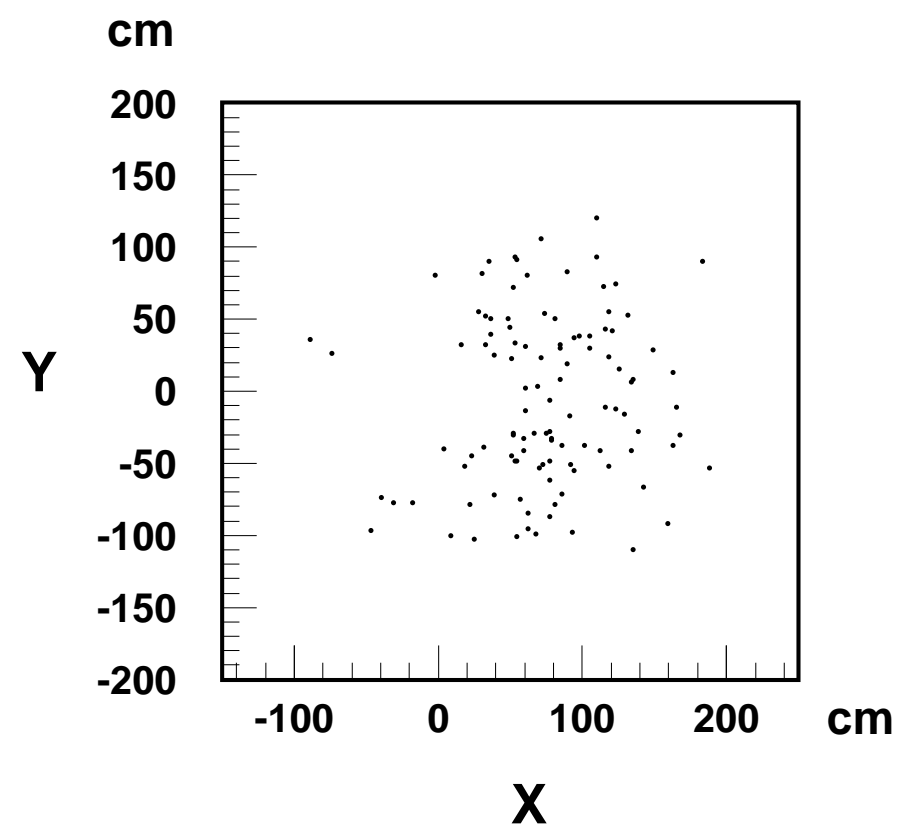

Fig. 4 
a)

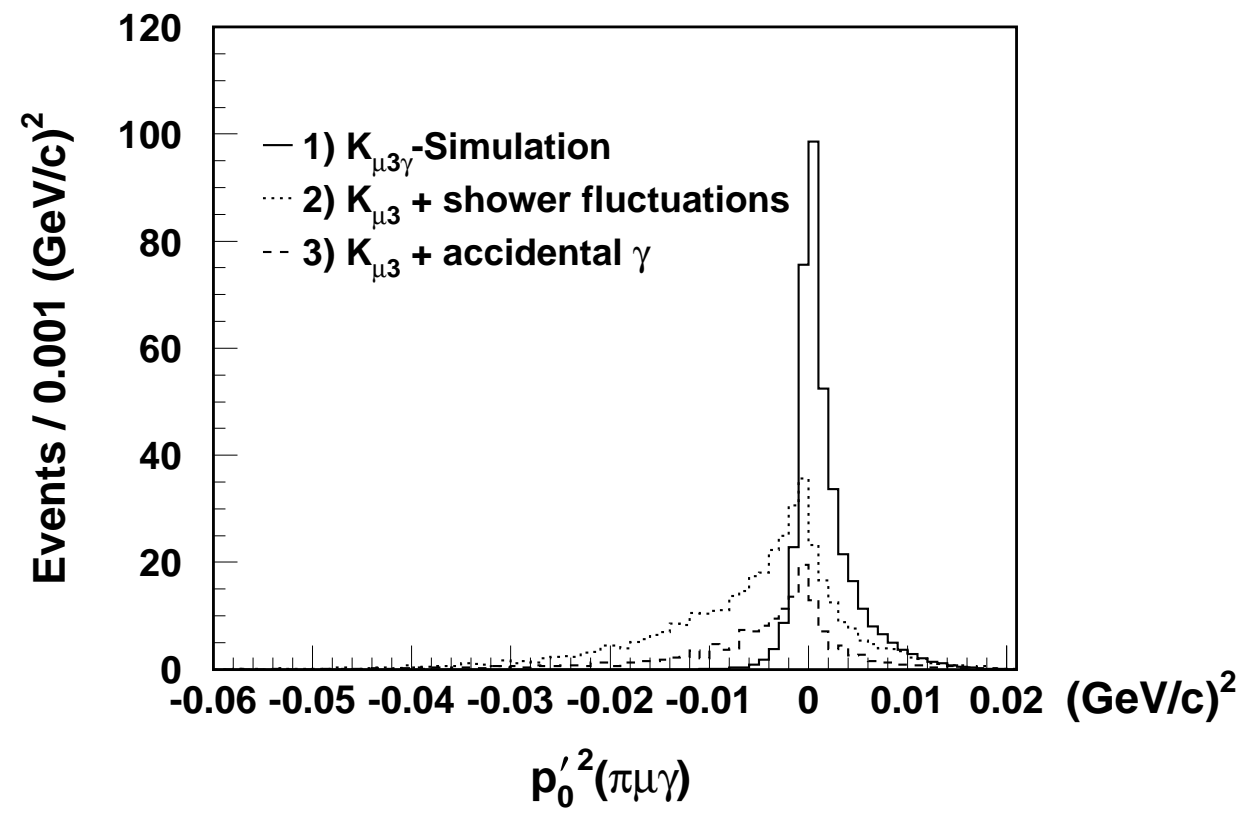

b)

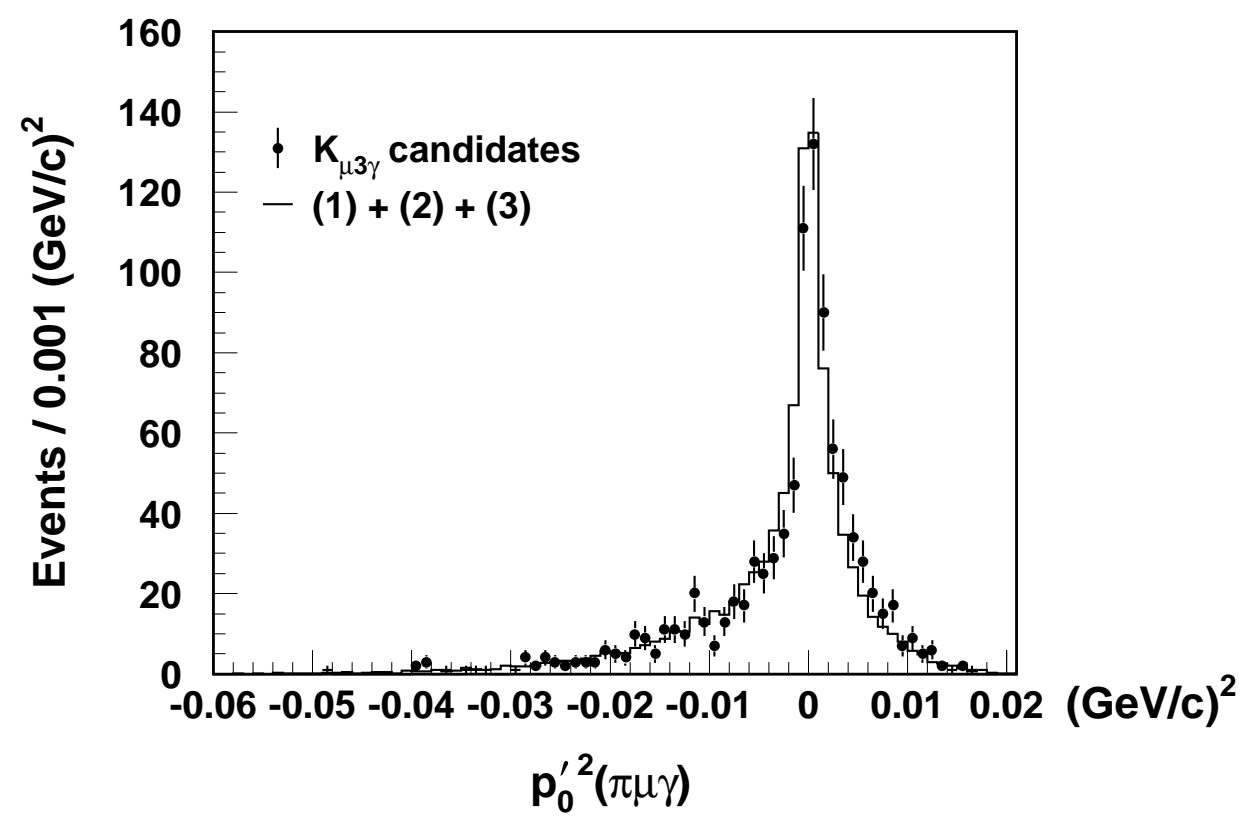

Fig. 5 
a)

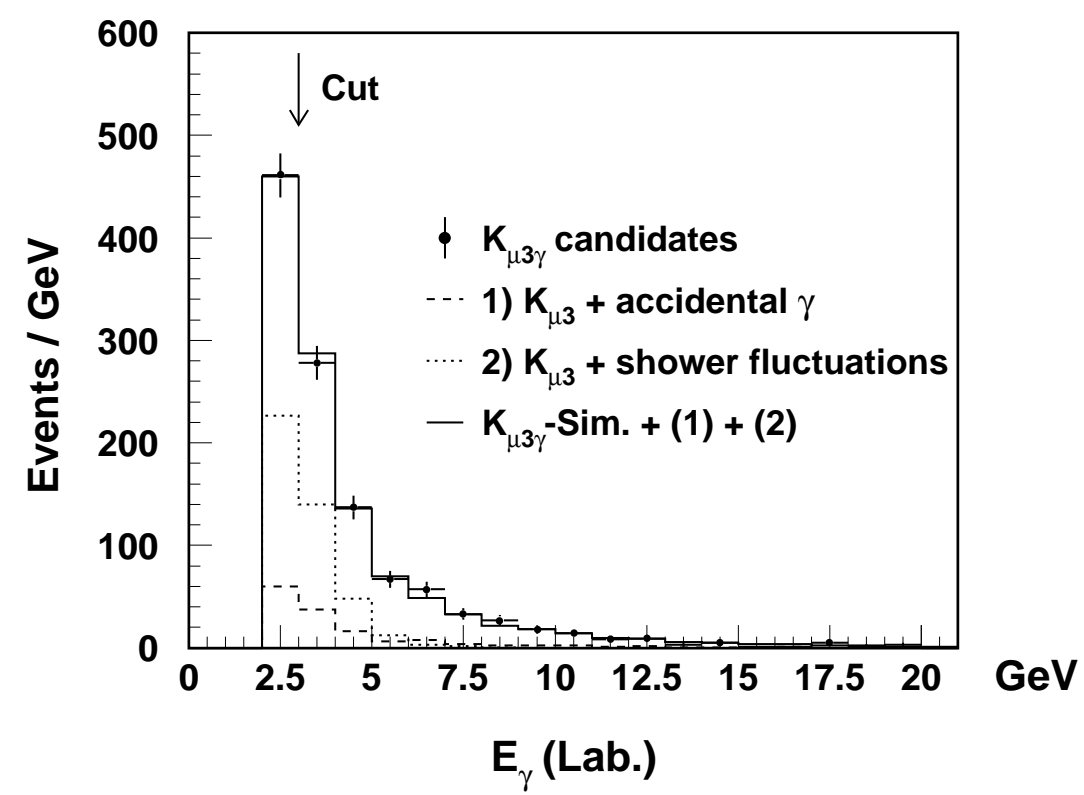

b)

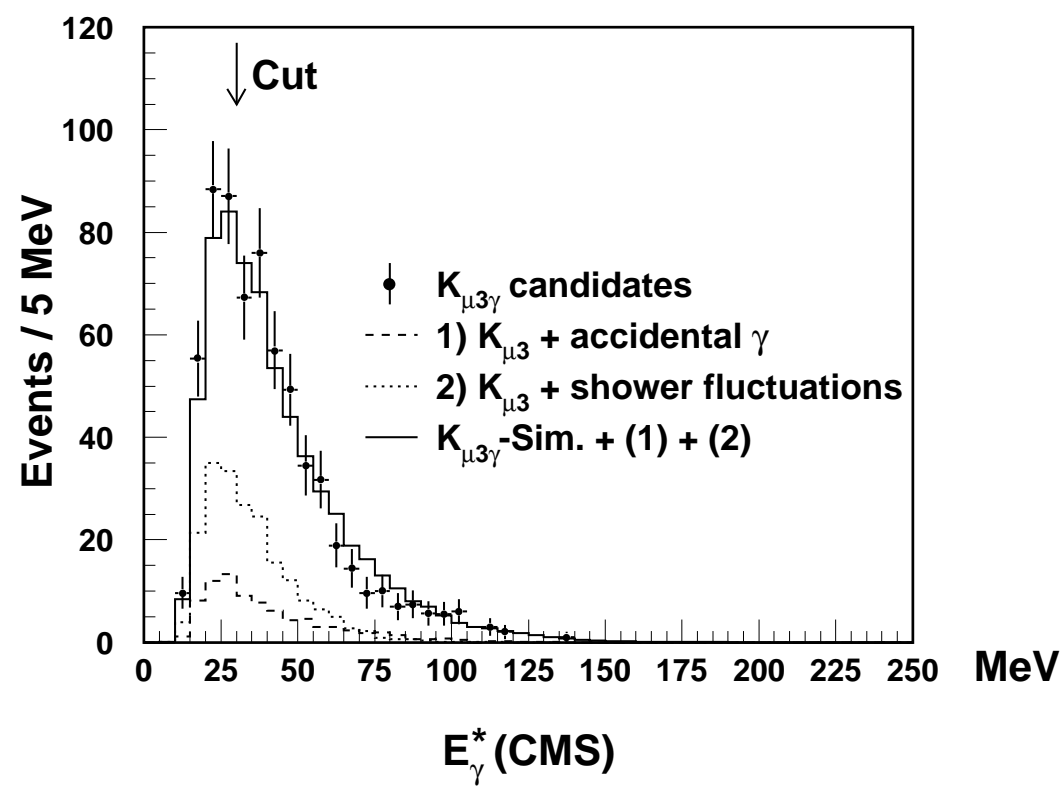

Fig. 6 